\title{
The at-risk registers in severe asthma (ARRISA) study: a cluster-randomised controlled trial examining effectiveness and costs in primary care
}

\author{
Jane Rebecca Smith, ${ }^{1}$ Michael J Noble, ${ }^{2}$ Stanley Musgrave, ${ }^{1}$ Jamie Murdoch, ${ }^{3}$ \\ Gill M Price, ${ }^{1}$ Garry R Barton, ${ }^{1}$ Jennifer Windley, ${ }^{2}$ Richard Holland, ${ }^{1}$ \\ Brian DW Harrison, ${ }^{1}$ Amanda Howe, ${ }^{1}$ David B Price, ${ }^{4}$ lan Harvey, ${ }^{1}$ \\ Andrew M Wilson ${ }^{1}$
}

- Additional materials are published online only. To view these files please visit the journal online (http://dx.doi.org/ 10.1136/thoraxjnl-2012202093).

${ }^{1}$ Norwich Medical School, University of East Anglia, Norwich, UK

${ }^{2}$ Acle Medical Centre, Acle, Norfolk, UK

${ }^{3}$ School of Nursing Sciences, University of East Anglia, Norwich, UK

${ }^{4}$ Research in Real Life Ltd Cawston, Norfolk, UK

Correspondence to Dr Jane Rebecca Smith, Norwich Medical School, University of East Anglia, Norwich NR4 7TJ, UK; j.r.smith@uea.ac.uk

Received 27 April 2012 Accepted 15 July 2012

Published Online First 31 August 2012

\section{ABSTRACT}

Background Patients at risk of severe exacerbations contribute disproportionally to asthma mortality, morbidity and costs. We evaluated the effectiveness and costs of using 'asthma risk registers' for these patients in primary care.

Methods In a cluster-randomised trial, 29 primary care practices identified 911 at-risk asthma patients using British asthma guideline criteria (severe asthma plus adverse psychosocial characteristics). Intervention practices added electronic alerts to identified patients' records to flag their at-risk status and received practicebased training about using the alerts to improve patient access and opportunistic management. Control practices continued routine care. Numbers of patients

experiencing the primary outcome of a moderate-severe exacerbation (resulting in death, hospitalisation, accident and emergency attendance, out-of-hours contact, or a course/boost in oral prednisolone for asthma), other healthcare and medication usage, and costs over 1 year were derived from practice-based records.

Results There was no significant effect on exacerbations (control: 46.5\%; intervention: $53.6 \%$, OR, $95 \% \mathrm{Cl} 1.30,0.93$ to 1.80). However, this composite outcome masked relative reductions in intervention patients experiencing hospitalisations (OR 0.50, 95\% Cl 0.26 to 0.94 ), accident and emergency (OR $0.74,95 \% \mathrm{Cl}$ 0.42 to 1.31 ) and out-of-hours contacts (OR 0.79, 95\% $\mathrm{Cl} 0.45$ to 1.37); and a relative increase in prednisolone prescription for exacerbations (OR 1.31, 95\% Cl 0.92 to 1.85). Furthermore, prescription of nebulised short-acting $\beta$-agonists reduced and long-acting $\beta$-agonists increased for intervention relative to control patients. The adjusted mean per patient healthcare cost was £138.21 lower $(p=0.837)$ among intervention practices.

Conclusion Using asthma risk registers in primary care did not reduce treated exacerbations, but reduced hospitalisations and increased prescriptions of recommended preventative therapies without increasing costs.

\section{INTRODUCTION}

Despite effective treatments and management guidelines, ${ }^{1}$ many asthma patients experience inadequate disease control, with asthma causing $1 \%$ of disability-adjusted life-years lost and 1 in 250

\section{Key messages}

What is the key question?

- Does implementing 'asthma risk registers' to flag the status of at-risk asthma patients in primary care, and providing training to practice staff on their use to facilitate access and opportunistic asthma management, reduce the number of at-risk patients experiencing moderate-severe exacerbations, improve aspects of care and reduce associated healthcare costs?

What is the bottom line?

- Using asthma risk registers in primary care did not reduce overall exacerbations. However, this composite outcome appeared to mask reductions in crisis events including hospitalisations alongside improvements in care reflected in increased prescriptions of recommended preventive therapies, without increasing overall healthcare costs.

\section{Why read on?}

- Use of 'asthma risk registers' is mentioned in British asthma guidelines, and this study is the first to detail how they can be implemented and provide reliable, generalisable evidence on their potential benefit.

deaths worldwide. ${ }^{2}$ Each year, $25 \%$ of asthma patients in Western Europe require unscheduled healthcare, and $7 \%$ are hospitalised, for asthma. ${ }^{3}$ Total annual societal costs of asthma are estimated at $\$ 56$ billion $^{4}$ and $£ 2.5$ billion $^{2}$ in the US and UK, respectively. Estimated costs of treating patients experiencing exacerbations are 3.5 times those of treating well-controlled asthma ${ }^{5}$ with unscheduled care accounting for over two-thirds of costs in those with poorly-controlled disease. ${ }^{6}$

Epidemiological studies ${ }^{7} 8$ suggest that the majority of patients at-risk of severe exacerbations are potentially identifiable. They characteristically have severe asthma plus poorly controlled disease, commonly resulting from poor adherence or other psychosocial problems. ${ }^{1}$ However, due to complicating clinical and psychosocial characteristics, these patients are often excluded from studies of, or fail to attend, ${ }^{9}$ educational initiatives that improve 
outcomes in general asthma populations. ${ }^{10}$ Existing evidence is thus unlikely to be generalisable to this group. Furthermore, there is a paucity of high-quality studies of non-pharmacological interventions specifically targeting patients with severe asthma, particularly in primary care. ${ }^{11}$

Although at-risk patients under-use primary care services and often fail to attend scheduled appointments, ${ }^{12}$ opportunistic asthma management and earlier emergency treatment might be facilitated if they are identified at each healthcare contact. Our pilot study using an 'asthma risk register' to alert staff to the status of at-risk asthma patients in a single primary care practice suggested it was a low-cost intervention that reduced emergency treatments and service use to levels of matched controls. ${ }^{13}$ Use of risk registers is mentioned in British asthma guidelines, ${ }^{1}$ but they are not included in UK quality measures for primary care ${ }^{14}$ due to lack of evidence. The aim of this study, conducted as a cluster-trial given the practice-level intervention, was therefore to evaluate whether, compared to routine care, use of asthma risk registers reduced numbers of at-risk patients experiencing exacerbations, improved aspects of care and altered associated healthcare costs.

\section{METHODS}

\section{Design and participants}

A pragmatic cluster-randomised trial in which data collected spanned the period November 2006-May 2009 was conducted in interested primary care practices in Norfolk, UK meeting the eligibility criteria of: (1) having a computer system that supported addition of electronic alerts to patient records; and (2) not already using an electronic system to support targeted, prospective management of selected at-risk asthma patients (figure 1). Clinicians at practices identified at-risk asthma patients aged $5+$ years in two stages using British guideline criteria $^{1}$ (figure 2). A database manager then randomised practices using a computer-generated list of random permutations with a block size of two and stratified according to whether their Index of Multiple Deprivation score was above or below the Norfolk median. ${ }^{15}$ Practices in a block were randomised simultaneously to ensure allocation concealment (ISRCTN trial register number 36918958).

\section{Intervention}

The practice-level intervention comprised:

1. Addition of electronic alerts visible to all staff to the computerised records of identified at-risk patients to flag their at-risk status at each contact. Wording was customised by the practice (eg, 'Asthma risk, prioritise care'; 'ARRISA: vulnerable asthmatic') and the appearance and visibility of alerts varied across different software (eg, pop-ups requiring active clearance; yellow post-it style notes remaining on screen).

2. A one hour practice-based training session (box 1) to support effective use of the alerts at which at least one representative from each staff group (GP, nurse, receptionist, manager/ administrator, dispenser) was present. The GP and nurse from the pilot study practice ${ }^{13}$ led the standardised training, which advised staff on how to engage with, and improve the routine and emergency management of, at-risk asthma patients using case examples to highlight potential actions for receptionists, clinicians and dispensary teams (box 1). Practices agreed an action plan for cascading information to absent staff, providing reminders for existing staff, and inducting new staff. Alerts were activated once dissemination was complete. Intervention practices had open access to the study team, were telephoned at 1,3 and 6 months and received a newsletter an average of 5 months after activation of alerts to reinforce implementation and encourage ongoing training. An
Figure 1 CONSORT diagram showing flow of practices $(\mathrm{N})$ and patients $(\mathrm{n})$ through the study.

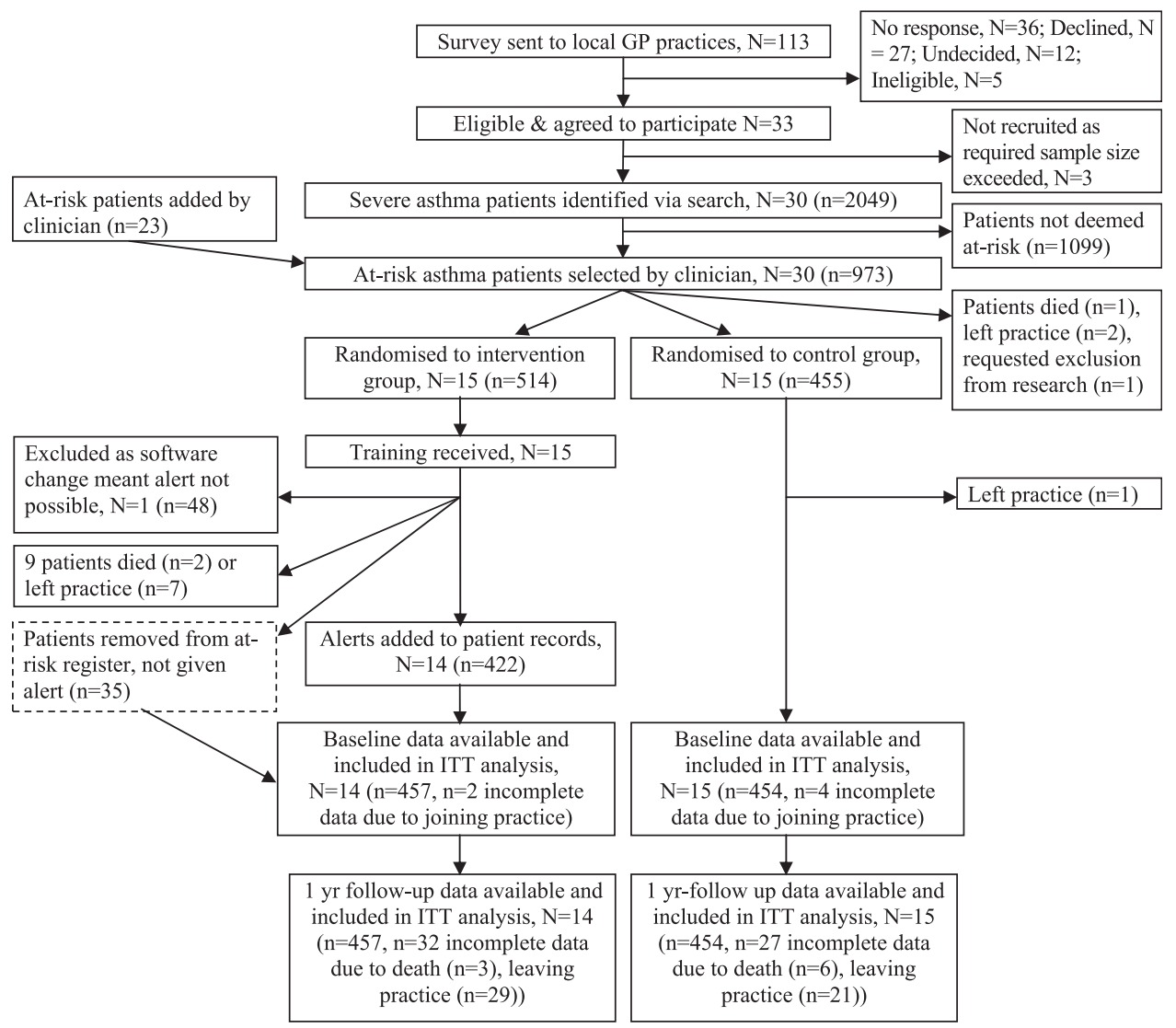


Figure 2 Identification and selection of patients for inclusion on asthma risk registers. ${ }^{*}$ This search had been piloted and optimised in an attempt to balance sensitivity and specificity at two different sized practices (9000 and 16900 patients) with differing software systems, at both identifying $9 \%$ of registered asthma patients as having severe asthma. †Due to poor coding of psychosocial problems in practice databases, electronic searches proved inadequate in identifying the majority of patients meeting criteria for being at-risk from their asthma at the pilot site $e^{13}$ so use of local clinical knowledge was essential to ensure comprehensive identification of at-risk patients.

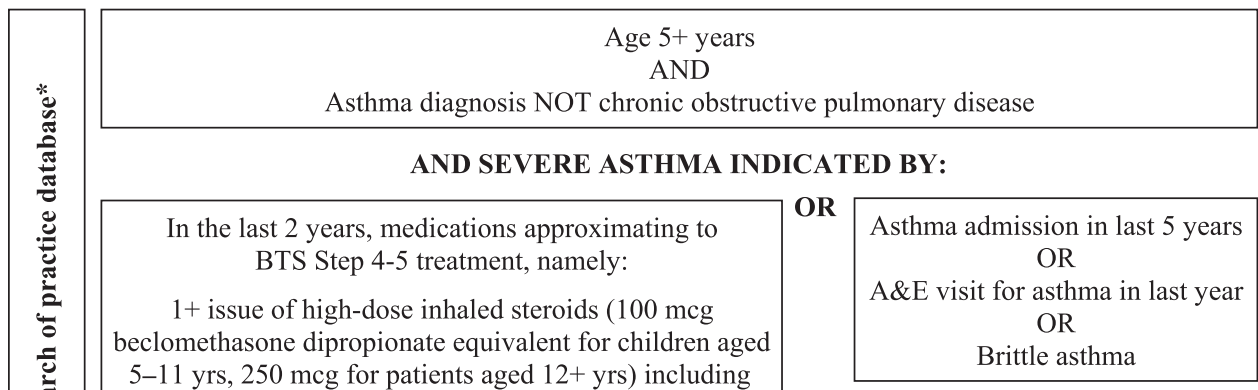

$5-11 \mathrm{yrs}, 250 \mathrm{mcg}$ for patients aged $12+\mathrm{yrs}$ ) including combination products AND

$1+$ issue of a long-acting beta-agonist $\mathrm{OR}$

leukotreine receptor antagonist OR

adverse reaction to a long-acting beta-agonist AND

1+ issue of prednisolone $5 \mathrm{mg}$ OR nebuliser

\section{AND PSYCHOSOCIAL PROBLEMS INDICATED BY:}

\begin{tabular}{|c|c|}
\hline & $\begin{array}{l}\text { Clinician opinion, with reference to patient notes as necess ary, regarding presence of one or more } \\
\text { of: } \\
\text { - Adherence problems } \\
\text { - Failure to attend primary or secondary care appointments or self-discharge from hospital } \\
\text { - Psychosis, depression, self-harm or other psychiatric illness } \\
\text { - Alcohol or drug abuse } \\
\text { - Major tranquiliser use } \\
\text { - Smoking } \\
\text { - Obesity } \\
\text { - Denial } \\
\text { - Learning difficulties } \\
\text { - Employment problems } \\
\text { - Social isolation } \\
\text { - Childhood abuse }\end{array}$ \\
\hline
\end{tabular}

OR

With justification and following discussion with the research team as necessary, other clinician-specified reason for patient not identified via above being considered at-risk from their asthma†. end-of-study questionnaire (online supplementary table A) assessed staff views on the intervention.

\section{Control practices}

Following identification of at-risk asthma patients, control practices continued usual care, comprising at least annual practice-based asthma reviews in nurse-led clinics, plus follow-up in secondary care outpatient clinics and emergency primary and secondary care for some patients as required. ${ }^{1}$

\section{Data collection and coding}

Anonymous patient-level data on primary care-based clinical events and medications were extracted electronically from practicebased computerised records using NHS MIQUEST ${ }^{16}$ or practicespecific software. Data on secondary care and out-of-hours service use were manually extracted from letters/reports in individual records. Baseline data were collected for the 1-year period prior to, and follow-up data for the 1-year period following, activation of alerts at intervention practices for each pair of practices randomised together. Data were imported/entered into an Access database. One of two researchers used all available information to code clinical events as associated with an exacerbation, otherwise respiratoryrelated or unrelated to asthma. Data were then processed to generate descriptive characteristics, outcomes, and resource use estimates. Validation of selected data with other sources (eg, for ambulance use) did not highlight any missing data in practice records and coding of deaths and exacerbations were checked (see below). Since electronically-extracted data did not always allow complete or accurate differentiation between GP and nurse, or respiratory and non-respiratory primary care contacts, detailed data on such contacts for costing were manually extracted from records for a random sample of up to five patients per practice.

The primary outcome was the number of patients experiencing a moderate-severe exacerbation, defined as per contemporaneous studies ${ }^{17}$ as those resulting in death (determined in a blinded review of records by two physicians), hospitalisation, accident and emergency (A\&E) attendance, out-of-hours medical contact, or a course or boost in oral corticosteroids (prednisolone) for asthma. An algorithm was applied to automatically code two or more events occurring $<14$ days apart as a single exacerbation. Where there was uncertainty regarding use of prednisolone for maintenance treatment versus exacerbations (based on three or more prescriptions of $>60$ prednisolone tablets), or three or more events were each separated by $<14$ days, prescription and clinical data were manually reviewed by researchers blind to study group and a clinical opinion sought 


\section{Box 1 Content of practice training sessions}

- Introductions

- Background and rationale covering evidence on morbidity and mortality and British guideline recommendations in relation to at-risk asthma patients; findings from the pilot study; the need to identify and facilitate rapid access for these patients; and the practice nurse perspective (to enhance nurse engagement and allay commonly expressed anxieties about increased workload).

- Study aims and design

- Interactive exploration of practice's existing experience, knowledge, awareness and current good practice with respect to at-risk asthma patients (eg, any known asthma deaths or example patients, associated risk factors, existing management strategies such as following up admissions/ out-of-hours contacts, prescription monitoring) and areas for improvement, which were probed to tailor subsequent discussions and the information provided.

- Case study-based discussion drawing from practice examples or three pre-prepared clinical cases highlighting issues and possible strategies for improved management for receptionists (eg, making a good first contact to improve engagement, fast-tracking clinical contact), clinicians (eg, being accessible, engaging and educating patients, and addressing psychosocial or other factors affecting asthma at every opportunity), and dispensary team (eg, being aware of prescription review dates and adherence indicators) as relevant to attendees.

- Discussion and agreement regarding practice-specific implementation covering:

- Appearance and wording of the electronic alert, which the practice customised to ensure optimal recognition and application.

- Proposed actions for different staff in response to the alerts.

- Parallel use of written lists in reception for some small practices.

- Agreement of a dissemination action plan, as necessary, for cascading information to all absent practice staff (who does, how done, use of reminders, informing new staff etc).

- The issue of disclosure to patients, which was left to the discretion of the practice after reviewing the study ethics approval and pilot study experiences.

- Amendments to the register, including questions regarding the eligibility of specific patients and forms to complete for adding or removing patients during the study so that all patients considered for inclusion could be tracked.

Follow-up plans

- Summary and thanks

Specific details of agreed implementation at the practice, along with generic content, were summarised in handouts provided to all attendees.

as necessary to determine the number of exacerbations represented. Exacerbation counts from a sub-sample of 50 such complex cases were compared between the two researchers and a further 20 were checked by an independent clinician, with high levels of agreement shown (Kappa for both $>0.71$ ).
Secondary outcomes were individual components comprising an exacerbation as above, outpatient attendances for asthma, primary care contacts, 'did not attends' (DNAs) at consultations, and asthma medications. Prescription data were processed to provide counts of inhalers/packs issued for medications, with combination products counted under both relevant categories and beclomethasone-equivalent daily doses calculated for inhaled corticosteroids. Asthma severity was described as the number of classes (0-9) of asthma medications prescribed in the baseline year, counting high- and low-dose inhaled corticosteroids separately. Smoking history, a Charlson co-morbidity index score, ${ }^{18}$ co-existing rhinitis, and socio-demographic characteristics were derived from baseline data, supplemented with follow-up data where this was missing.

\section{Statistical analyses}

An effective sample size of 134 patients per group was initially calculated assuming Type I error 0.05, Type II error 0.1, and an exacerbation rate of $50 \%$ in controls and $30 \%$ in the intervention group, based on pre- and post-intervention observations in the pilot study. ${ }^{13}$ To adjust for clustering, a mean cluster size (number of at-risk asthma patients per practice) of 20 and an intra-cluster correlation coefficient (ICC) of $0.05,{ }^{19}$ was assumed, yielding a sample size of 268 patients per group. However, analyses of baseline exacerbation data from the initial 19 practices recruited indicated a mean cluster size of 36 , and an ICC $=0.068$ which, with other previous assumptions, suggested randomising 453 patients (13 practices) per group to achieve the equivalent of the original sample size based on individual randomisation.

Descriptive data were visually compared to identify any major baseline differences between groups, and covariates for inclusion in fully adjusted analyses were selected from a list of pre-specified prognostic variables. Adjustments were made for independent predictors of the primary outcome ${ }^{20}$ identified via simple and multiple regression.

Mixed-effect models were used to adjust for clustering of outcomes within practices ${ }^{21}$ in producing effect sizes, $95 \%$ $\mathrm{CIs}$ and $\mathrm{p}$ values. Analyses were conducted with random effect for practice, adjusted for stratification (above/below-average Index of Multiple Deprivation) alone ('unadjusted') and additionally adjusted for baseline values of the outcome and other selected covariates ('adjusted'). Random-effects logistic models producing ORs were used for binary outcomes (n, \%) since there was no difference in follow-up times between groups $(p=0.458$; Mann-Whitney test). ICCs were estimated in these models for the primary outcome and its components. Random-effects negative-binomial models, producing rate-ratios (RRs), taking into account each patient's observation time, were used for outcomes experienced by the majority of patients where ORs exaggerate effects. Results for these were described using median and IQR rates (counts per year), since they were generally heavily positively skewed with extra-Poisson variation.

The main analyses were undertaken on an intentionto-treat (ITT) basis including all at-risk patients identified prerandomisation who were alive and registered with practices on the date alerts were activated at intervention practices. Additional analyses were conducted in a 'pure' sub-group of the ITT sample (analogous to 'per protocol') that excluded patients: (1) removed from registers by practices between randomisation and the start of follow-up, whose records therefore did not receive an alert, or (2) subsequently identified as having a COPD diagnosis recorded during their baseline year. To examine potential impacts of variations in the visibility of alerts and data extraction methods, another pre-specified, secondary ITT 
analysis tested the interaction between practice software-type (EMIS vs non-EMIS) and the intervention. Analyses were performed using Stata (StataCorp. Stata Statistical Software: Release V.11.1SE, 2009). Nominal significance level was 5\% throughout; with no adjustments for multiple testing.

\section{Cost analyses}

Total respiratory- and non-respiratory-related costs were estimated from the viewpoint of the UK NHS with resource use valued at 2007-2008 rates using unit costs from published ${ }^{22-25}$ or local sources as applicable. Implementation costs (searching for and identifying patients, setting up alerts, delivering training, and practice follow-up) for each practice were estimated using researcher records. Per-patient costs for the entire baseline and follow-up years were estimated and for patients joining practices during baseline or leaving during follow-up the average cost per day for items in the same period was applied to any time for which data were missing.

The mean change in annual NHS costs was estimated for control and intervention groups. Additionally, the (unadjusted)

Table 1 Baseline practice and patient characteristics

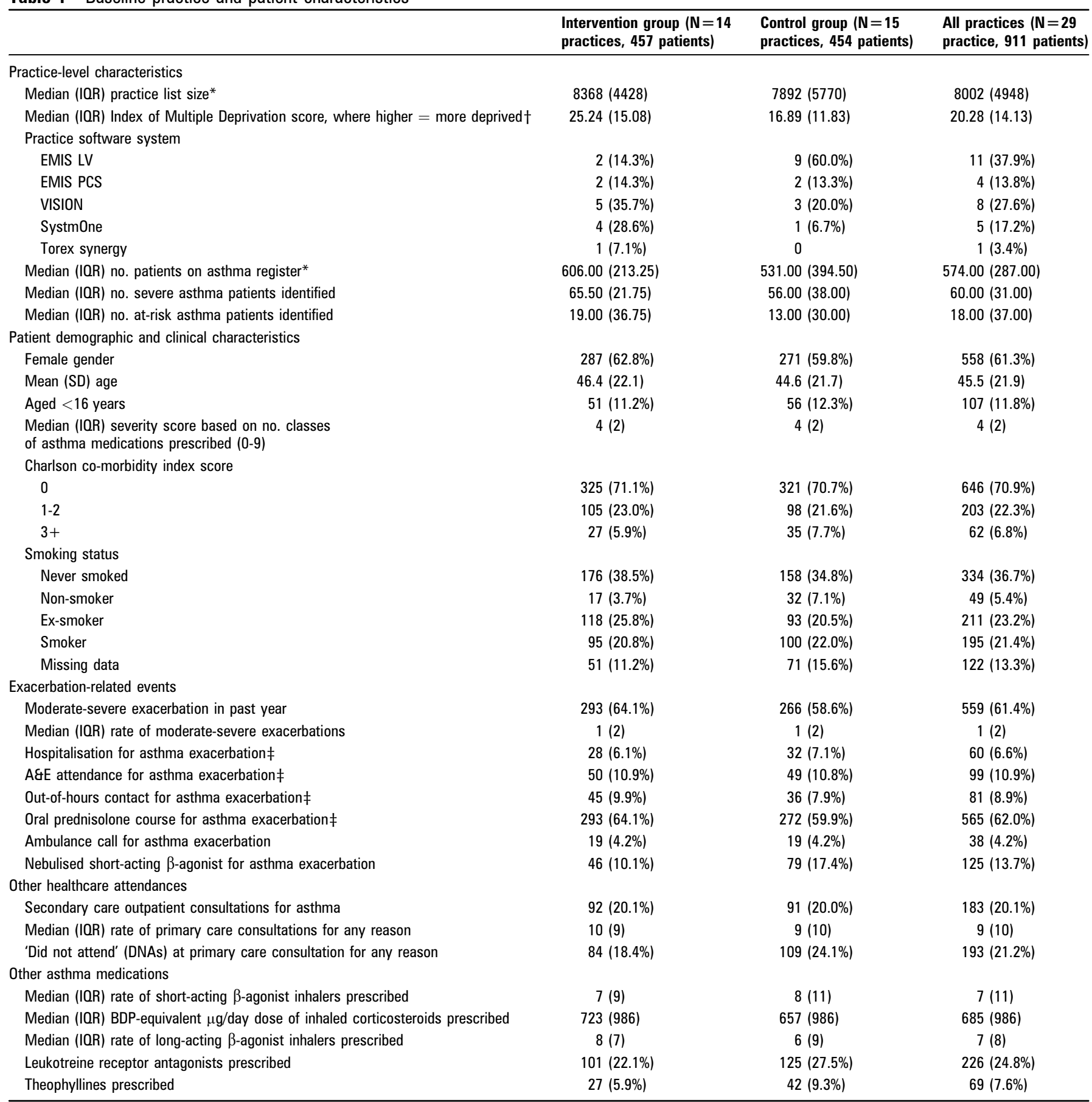

Data are $\mathrm{N}(\%)$ and reported at patient-level unless otherwise indicated.

*Source: 0OF0708_Pracs_Prevalence.xls spreadsheet via http://www.ic.nhs.uk/statistics-and-data-collections/audits-and-performance/the-quality-and-outcomes-framework/the-quality-andoutcomes-framework-2007-08.

†Source: Norfolk Deprivation data 2007 provided by NHS Norfolk, see: http://www.norfolkinsight.org.uk/deprivation for further information.

$\neq$ Event included in count of exacerbations (see methods for full definition). 
mean incremental cost was estimated by taking the difference between the mean change in costs across groups. Finally, to take account of clustering, a two-stage bootstrap procedure was used to estimate the adjusted incremental cost between groups. ${ }^{26}$

\section{RESULTS}

Thirty practices were recruited. One intervention practice was excluded after training due to a software change precluding activation of alerts. Twenty-nine practices therefore identified 911 at-risk asthma patients with data available for analyses (figure 1). Baseline characteristics (table 1) were similar, except that at practice level intervention practices had larger and more deprived populations, and were less likely using EMIS software. There were some differences in patient-level exacerbations and DNA rates, and in medications prescribed, especially nebulised bronchodilators. Adjustments were made for baseline differences in prognostic variables as indicated.

Mean observation periods (table 2) and losses to follow-up through patients dying or leaving practices (figure 1) were similar across groups $(93.0 \%$ intervention, $94.7 \%$ control with complete data). Nine patients died during follow-up, six of these (including the only one deemed due to asthma) were in the control group.

In unadjusted and fully adjusted ITT analyses, there was no significant difference between groups in the number of patients experiencing a moderate-severe exacerbation (primary outcome expressed as odds) during the follow-up year (table 2) or in the rate of exacerbations per person per year (median $(I O R)=1(2)$ for intervention, 0 (2) for control; RR (95\% CI) 1.21 (0.95 to 1.55), $\mathrm{p}=0.13$ unadjusted; 1.16 (0.97 to 1.38$), \mathrm{p}=0.11$ adjusted). However, disaggregating this composite outcome, the intervention halved the odds of patients experiencing hospitalisation, nonsignificantly reduced the proportions using $A \& E$ and out-of-hours services and increased the odds of patients receiving pulse prednisolone. Significantly fewer intervention patients received nebulised $\beta$-agonists and they were prescribed significantly more long-acting beta-agonists (table 2). Findings were similar when analyses were confined to the 'pure group' excluding intervention group patients who did not receive alerts $(n=35)$ or were found to have a COPD diagnosis $(n=24)$ (online supplementary table $B$ ). Similar patterns and sizes of effects were also observed when rates of events were analysed to take account of truncated follow-up for some patients. Software-type was not related to the primary outcome and there were no significant interactions of softwaretype with the intervention for the primary outcome (OR (95\% $\mathrm{CI}$ ) for unadjusted interaction $=1.17$ ( 0.57 to 2.38 ); $\mathrm{p}=0.7$ ). The ICC for the primary outcome, conditional on stratification, was 0.013 (95\% CI 0.002 to 0.087 ). ICCs for components of this outcome are shown in online supplementary table C.

\section{Costs}

The mean per practice intervention implementation cost was $£ 1687.40$, comprising set-up (£414.24), training (£1211.17) and follow-up ( $£ 62.00$ ), equating to an average of $£ 51.69$ per patient. When proportions of nurse $(41.2 \%, \mathrm{n}=1070)$, GP $(58.8 \%, \mathrm{n}=1524)$, respiratory-related $(34.2 \%, \mathrm{n}=887)$ and

Table 2 Primary and secondary outcomes at 1 year: results from intention-to-treat analyses

\begin{tabular}{|c|c|c|c|c|c|c|}
\hline & $\begin{array}{l}\text { Intervention } \\
(\mathrm{N}=14 \text { pracs, } \\
\mathrm{n}=457 \mathrm{px})\end{array}$ & $\begin{array}{l}\text { Control } \\
(\mathrm{N}=15 \text { pracs, } \\
\mathrm{n}=454 \mathrm{px} \text { ) }\end{array}$ & $\begin{array}{l}\text { Unadjusted } \\
\text { OR* }(95 \% \mathrm{CI}) \\
\text { unless indicated }\end{array}$ & $\mathbf{p}$ & $\begin{array}{l}\text { Adjusted } \\
\text { OR } †(95 \% \text { CI) } \\
\text { unless indicated }\end{array}$ & p Value \\
\hline $\begin{array}{l}\text { Mean (range) cluster size, comprising number of high risk } \\
\text { patients on register per practice }\end{array}$ & $32.6(3 ; 82)$ & $30.3(4 ; 90)$ & & & & \\
\hline Mean (SD) days per person post-intervention observation time & $352(54)$ & $358(36)$ & & & & \\
\hline \multicolumn{7}{|l|}{ Primary outcome } \\
\hline Moderate-severe asthma exacerbation & $245(53.6 \%)$ & $211(46.5 \%)$ & $1.30(0.93$ to 1.80$)$ & 0.120 & $1.27(0.95$ to 1.70$)$ & 0.105 \\
\hline \multicolumn{7}{|l|}{ Secondary outcomes: exacerbation-related events } \\
\hline Hospitalisation for asthma exacerbation $\ddagger$ & $15(3.3 \%)$ & $29(6.4 \%)$ & $0.50(0.26$ to 0.94$)$ & 0.031 & $0.51(0.26$ to 1.00$)$ & 0.051 \\
\hline A\&E attendance for asthma exacerbation $\ddagger$ & $29(6.4 \%)$ & $37(8.2 \%)$ & $0.74(0.42$ to 1.31$)$ & 0.303 & $0.70(0.37$ to 1.34$)$ & 0.284 \\
\hline Out-of-hours contact for asthma exacerbation $\ddagger$ & $26(5.7 \%)$ & $32(7.1 \%)$ & $0.79(0.45$ to 1.37$)$ & 0.396 & $0.75(0.41$ to 1.37$)$ & 0.350 \\
\hline Oral prednisolone course for asthma exacerbation $\ddagger$ & $247(54.1 \%)$ & $213(46.9 \%)$ & $1.31(0.92$ to 1.85$)$ & 0.136 & $1.28(0.95$ to 1.73$)$ & 0.112 \\
\hline Ambulance call for asthma exacerbation & $13(2.8 \%)$ & $12(2.6 \%)$ & $1.03(0.42$ to 2.51$)$ & 0.950 & $1.02(0.45$ to 2.35$)$ & 0.954 \\
\hline Nebulised short-acting $\beta$-agonist for asthma exacerbation & $36(7.9 \%)$ & $63(13.9 \%)$ & $0.51(0.29$ to 0.88$)$ & 0.016 & $0.61(0.36$ to 1.02$)$ & 0.061 \\
\hline \multicolumn{7}{|l|}{ Secondary outcomes: other healthcare attendances } \\
\hline Secondary care outpatient consultations for asthma & $81(17.7 \%)$ & $71(15.6 \%)$ & $1.14(0.76$ to 1.70$)$ & 0.532 & $1.27(0.82$ to 1.98$)$ & 0.283 \\
\hline $\begin{array}{l}\text { Median (IQR) rate of primary care consultations } \\
\text { for any reason }\end{array}$ & $9(11)$ & $8(11)$ & $1.09 \S(0.95$ to 1.26$)$ & 0.234 & $1.069(0.97$ to 1.16$)$ & 0.190 \\
\hline $\begin{array}{l}\text { 'Did not attend' (DNAs) at primary care consultation } \\
\text { for any reason }\end{array}$ & $82(17.9 \%)$ & $105(23.1 \%)$ & $0.64(0.29$ to 1.42$)$ & 0.270 & $0.73(0.35$ to 1.52$)$ & 0.396 \\
\hline \multicolumn{7}{|l|}{ Secondary outcomes: other asthma medications } \\
\hline Median (IOR) rate of short-acting $\beta$-agonist inhalers prescribed & $6(10)$ & $7(12)$ & $1.03 \S(0.89$ to 1.19$)$ & 0.666 & $1.039(0.91$ to 1.17$)$ & 0.600 \\
\hline $\begin{array}{l}\text { Median (IQR) BDP-equivalent } \mu \mathrm{g} / \text { day dose of inhaled } \\
\text { corticosteroids prescribed }\end{array}$ & $658(1036)$ & $658(1036)$ & $1.10 \S(0.97$ to 1.25$)$ & 0.152 & $1.07 \Phi(0.96$ to 1.18$)$ & 0.223 \\
\hline Median (IQR) rate of long-acting $\beta$-agonist inhalers prescribed & $8(9)$ & $6(9)$ & $1.24 \S(1.08$ to 1.43$)$ & 0.002 & $1.08 \uparrow(0.99$ to 1.17$)$ & 0.085 \\
\hline Leukotreine receptor antagonists prescribed & $111(24.3 \%)$ & $131(28.9 \%)$ & $0.85(0.55$ to 1.31$)$ & 0.459 & $0.96(0.49$ to 1.86$)$ & 0.895 \\
\hline Theophyllines prescribed & $23(5.0 \%)$ & $40(8.8 \%)$ & $0.56(0.29$ to 1.07$)$ & 0.079 & $0.53(0.21$ to 1.34$)$ & 0.179 \\
\hline
\end{tabular}

Data are at patient-level, showing $\mathrm{N}(\%)$ experiencing event per year and ORs unless otherwise indicated.

*OR estimated using 'univariate' analysis of proportions in random-effects logistic regression model, adjusted for clustering and stratification only.

†OR estimated using multivariable analysis of proportions in random-effects logistic regression model, adjusted for clustering and stratification plus baseline values of outcome variable, exacerbation count, age (continuous), asthma severity (no. classes of meds), Charlson co-morbidity index (in 3 categories), and rhinitis.

‡Event included in count of exacerbations (see methods for full definition).

$\S$ Rate ratio estimated using 'univariate' analysis of counts in random-effects negative binomial regression model, adjusted for clustering and stratification only.

TRate ratio estimated using multivariable analysis of counts in random-effects negative binomial regression model adjusted for clustering and stratification plus baseline values of outcome variable, exacerbations count, age (continuous), asthma severity (\#classes of meds), Charlson co-morbidity index (in 3 categories), and rhinitis.

A\&E, accident and emergency. 
non-respiratory-related visits $(65.8 \%, \mathrm{n}=1707)$ obtained from manual extraction of data on 2594 visits from a sub-sample of 139 patients were applied to all primary care contacts, mean primary care costs were estimated to be similar in both groups during baseline and follow-up (table 3). Estimated medication costs were also similar. Out-of-hours costs tended to be lower during follow-up for both groups, whereas secondary care costs tended to be higher (mean change estimates favoured the intervention group for both). Overall, there was an increase in costs for both groups but the increase was larger for the control group resulting in an overall (unadjusted) incremental cost of -£88.91 per patient (table 3) that is, mean NHS costs were lower in intervention compared to control practices despite the additional $£ 52$ cost of implementing the intervention. When clustering was accounted for the adjusted mean per patient cost was estimated as $-£ 138.21$ (95\% CI $-£ 1248.36$ to $£ 910.58, p=0.837$ ).

\section{End of study questionnaire}

Eleven of 14 intervention practices returned questionnaires, with at least two categories of staff (GP, nurse, receptionist) responding from each. Results (online supplementary table A) suggest almost all staff felt the alerts were useful to at least some extent and would continue using them, most felt they did not increase workload and negative comments were received from only one practice. Despite this, $70 \%$ felt that the alerts had altered their management only slightly or not at all.

\section{DISCUSSION \\ Summary of findings}

Establishing electronic alerts linked to asthma risk registers and providing training on their use to facilitate access and provision of opportunistic asthma care did not reduce a composite indicator of moderate-severe exacerbations using contemporaneous ${ }^{17}$ or current definitions. ${ }^{27}$ However, these definitions include an indicator of good practice (prescription of prednisolone for exacerbations) alongside adverse outcomes (A\&E attendances, hospitalisations), and potentially mask the benefits of this type of intervention, particularly for patients with severe asthma experiencing frequent exacerbations. Considering this, we showed reductions in hospitalisations and nebulised $\beta$-agonists prescribed; and increases in prescription of preventive therapies as per guidelines that is, oral prednisolone for exacerbations and long-acting beta-agonists for patients already on inhaled corticosteroids. There were also non-significant reductions in $\mathrm{A} \& \mathrm{E}$ attendances and DNAs for routine appointments. Although numerous endpoints were examined with only a few secondary outcomes showing consistently significant findings, the overall pattern of results suggest that use of asthma risk registers improved patient management (eg, more appropriate prescribing) and patient outcomes (reduced crisis events, including hospitalisations). Furthermore, the intervention was generally well-accepted and did not increase costs.

\section{Interpretation in light of previous research}

Hospitalisation represents a significant event in the lives of asthma patients, is an indicator of severity, predicts re-admission and death ${ }^{28} 29$ and constitutes a major expense. ${ }^{4} 6$ Identifying methods for reducing hospitalisations is therefore paramount to patients, healthcare providers and funders. Although small numbers $(7 \%)$ experienced hospitalisations, our intervention reduced admissions by a similar magnitude to that observed across more intensive asthma education programmes, ${ }^{10}$ including among children $^{30}$ or adults attending A\&E for asthma. ${ }^{31}$ Poor adherence, including failing to attend appointments, is common among at-risk patients ${ }^{7} 9$ and notably only $20 \%$ of our sample attended secondary care asthma clinics, highlighting the importance of primary care intervention.

Asthma risk registers represent a complex intervention and any beneficial effects may stem from impacts of the alerts and

Table 3 Per patient mean annual levels of resource use and associated estimated costs in each group and unadjusted incremental costs

\begin{tabular}{|c|c|c|c|c|c|c|c|c|c|c|c|c|c|}
\hline \multirow[b]{2}{*}{ Item } & \multirow[b]{2}{*}{ Group } & \multicolumn{4}{|c|}{ Respiratory related } & \multicolumn{4}{|c|}{ Non-respiratory related } & \multicolumn{4}{|l|}{ Overall } \\
\hline & & Baseline & Follow-up & Change & $\begin{array}{l}\text { Incremental } \\
\text { cost }\end{array}$ & Baseline & Follow-up & Change & $\begin{array}{l}\text { Incremental } \\
\text { cost }\end{array}$ & Baseline & Follow-up & Change & $\begin{array}{l}\text { Incrementa } \\
\text { cost }\end{array}$ \\
\hline \multirow[t]{2}{*}{ Intervention cost } & Intervention & - & $£ 51.69$ & $£ 51.69$ & $£ 51.69$ & - & - & - & & - & $£ 51.69$ & $£ 51.69$ & $£ 51.69$ \\
\hline & Control & - & - & - & & - & - & - & & - & - & - & \\
\hline \multicolumn{14}{|l|}{ Primary care } \\
\hline \multirow[t]{2}{*}{ No. contacts } & Intervention & 4.06 & 3.92 & -0.14 & & 7.83 & 7.56 & -0.26 & & 11.88 & 11.48 & -0.40 & \\
\hline & Control & 3.98 & 3.80 & -0.18 & & 7.68 & 7.33 & -0.35 & & 11.66 & 11.12 & -0.54 & \\
\hline \multirow[t]{2}{*}{ Cost } & Intervention & $£ 104.39$ & $£ 100.88$ & $-£ 3.51$ & $£ 1.21$ & $£ 200.89$ & $£ 194.13$ & $-£ 6.76$ & $£ 2.33$ & $£ 305.28$ & $£ 295.01$ & $-£ 10.27$ & $£ 3.54$ \\
\hline & Control & $£ 102.42$ & $£ 97.70$ & $-£ 4.72$ & & $£ 197.11$ & $£ 188.02$ & $-£ 9.09$ & & $£ 299.54$ & $£ 285.73$ & $-£ 13.81$ & \\
\hline \multicolumn{14}{|l|}{ Secondary care } \\
\hline \multirow[t]{2}{*}{ No. contacts } & Intervention & 0.86 & 0.63 & -0.23 & & 2.65 & 2.56 & -0.09 & & 3.51 & 3.19 & -0.32 & \\
\hline & Control & 0.89 & 0.67 & -0.22 & & 2.49 & 2.34 & -0.15 & & 3.38 & 3.00 & -0.37 & \\
\hline \multirow[t]{2}{*}{ Cost } & Intervention & $£ 250.68$ & $£ 274.97$ & $£ 24.29$ & $£ 3.60$ & $£ 673.13$ & $£ 684.98$ & $£ 11.85$ & $-£ 121.52$ & $£ 923.81$ & $£ 959.95$ & $£ 36.14$ & $-£ 117.93$ \\
\hline & Control & $£ 322.29$ & $£ 342.99$ & $£ 20.70$ & & $£ 700.84$ & $£ 834.21$ & $£ 133.37$ & & $£ 1023.13$ & $£ 1177.20$ & $£ 154.07$ & \\
\hline \multicolumn{14}{|l|}{ Out of hours } \\
\hline \multirow[t]{2}{*}{ No. contacts } & Intervention & 0.26 & 0.16 & -0.09 & & 0.26 & 0.17 & -0.09 & & 0.52 & 0.34 & -0.18 & \\
\hline & Control & 0.27 & 0.28 & 0.01 & & 0.54 & 0.39 & -0.15 & & 0.81 & 0.67 & -0.14 & \\
\hline \multirow[t]{2}{*}{ Cost } & Intervention & $£ 10.83$ & $£ 7.49$ & $-£ 3.34$ & $-£ 3.71$ & $£ 10.46$ & $£ 6.28$ & $-£ 4.18$ & $£ 2.34$ & $£ 21.29$ & $£ 13.76$ & $-£ 7.52$ & $-£ 1.37$ \\
\hline & Control & $£ 11.09$ & $£ 11.46$ & $£ 0.37$ & & $£ 20.58$ & $£ 14.07$ & $-£ 6.52$ & & $£ 31.67$ & $£ 25.52$ & $-£ 6.15$ & \\
\hline \multicolumn{14}{|l|}{ Medication } \\
\hline \multirow{2}{*}{$\begin{array}{l}\text { No. } \\
\text { prescriptions }\end{array}$} & Intervention & 25.67 & 17.82 & -7.85 & & 38.59 & 37.74 & -0.85 & & 64.26 & 55.56 & -8.70 & \\
\hline & Control & 19.76 & 18.75 & -1.00 & & 39.93 & 42.68 & 2.75 & & 59.68 & 61.43 & 1.75 & \\
\hline \multirow[t]{2}{*}{ Cost } & Intervention & $£ 488.62$ & $£ 488.49$ & $-£ 0.13$ & $£ 9.24$ & $£ 390.82$ & $£ 381.14$ & $-£ 9.68$ & $-£ 34.08$ & $£ 879.45$ & $£ 869.64$ & $-£ 9.81$ & $-£ 24.84$ \\
\hline & Control & $£ 489.25$ & $£ 479.88$ & $-£ 9.37$ & & $£ 377.09$ & $£ 401.48$ & $£ 24.40$ & & $£ 866.33$ & $£ 881.36$ & $£ 15.03$ & \\
\hline \multirow[t]{2}{*}{ Total cost } & Intervention & $£ 854.52$ & $£ 923.52$ & $£ 69.00$ & $£ 62.03$ & $£ 1275.31$ & $£ 1266.53$ & $-£ 8.77$ & $-£ 150.94$ & $£ 2129.83$ & $£ 2190.06$ & $£ 60.23$ & $-£ 88.91$ \\
\hline & Control & $£ 925.06$ & $£ 932.03$ & $£ 6.97$ & & $£ 1295.62$ & $£ 1437.79$ & $£ 142.17$ & & $£ 2220.68$ & $£ 2369.81$ & $£ 149.14$ & \\
\hline
\end{tabular}


training on enhanced access, improved routine and emergency care and/or increased opportunistic asthma management. However, feedback from staff suggests that despite recognising the utility of the registers, they perceived few impacts on their management, including the ability of receptionists to prioritise appointments. The mechanisms of any effects therefore remain unexplored, though they were not dependent on practice software-type and nature of the alerts and likely stem from a combination of the training and electronic flagging of at-risk patients. Patient education is considered essential to asthma management, but health professional education has been less well-investigated. ${ }^{32}$ However, in the more intensive ELECTRA study intervention, ${ }^{33}$ review of at-risk asthma patients in a nurseled clinic, and educational outreach and promotion of guidelines for at-risk asthma to primary care clinicians delayed time to first presentation and reduced the proportion of at-risk patients attending with exacerbations (OR 0.62 , CI 0.83 to 1.01 ).

\section{Strengths and limitations}

Variability in the characteristics of practices and their implementation of the intervention may explain the wide CIs around estimates of effects and costs, but also increases generalisability of the findings. Use of anonymous, routinely available data to assess outcomes ensures applicability of findings to all at-risk asthma patients. However, it failed to provide a more patientfocussed outcome suitable for use in a full cost-effectiveness analysis and there were some difficulties with data extraction. Practice software did not permit accurate or comprehensive determination of whether primary care attendances were nurse or GP-led, or related to asthma. Consequently, data were manually extracted for a sub-sample to inform the primary care resource utilisation and costs, though this represented a small proportion of total costs. Incomplete coding of hospitalisations and $A \& E$ attendances in practice records also meant there was potential for some exacerbations treated only in hospital to be missed. However, $86 \%$ of all exacerbations identified were associated with a prescription of prednisolone in primary care, which is comprehensively coded, so numbers are likely to be small and to have had minimal impact on results since control and intervention groups would have been similarly affected. We also undertook manual review of clinical information and selected blind checks for the $15 \%$ of patients for whom the number of exacerbations was uncertain, manual review of consultation data for up to five patients per practice, and validation with other sources where possible to maximise the accuracy and completeness of our data.

\section{CONCLUSIONS AND IMPLICATIONS}

Our findings suggest potential clinical benefit for a group at serious risk from their asthma, who utilise a high proportion of costly healthcare resources and, as a result of psychosocial problems, often have difficulty engaging with healthcare services and interventions. The use of registers for these patients may reduce hospitalisations and improve aspects of their care, such as prescribing practice, with no increase in perceived staff workload or costs. This study does not provide conclusive evidence to support the suggestion in current British asthma guidelines ${ }^{1}$ that asthma risk registers may be helpful. However, it informs how they can be implemented in practice and provides a basis for further research studies.

Acknowledgements With thanks to Asthma UK for funding, all the practices and their staff involved, patient representatives on the Project Management Group
Penny Vicary and Margaret Kent, Dr Shuaib Nasser, Dr Richard Gilbert and Dr John Battersby who served on the Independent Trial Steering Committee, and Database Manager Tony Dyer, Computer Science student Ricardo Ramirez-Gonzalez and Researcher Adam Martin for database development and assistance with database programming and costing respectively.

Contributors All authors had full access to all of the data (including statistical reports and tables) in the study and can take responsibility for the integrity of the data and the accuracy of the data analysis. JRS was a joint principal investigator. She was involved in the pilot study, contributed to the original protocol design, led the funding application and co-ordinated the overall running of the project. She oversaw the outcome and cost analyses, contributed to interpretation of findings and drafted and edited the paper. MJN led the pilot study, contributed to protocol design, led the design and delivery of the intervention, advised on data collection and coding, contributed to interpretation of findings, drafted sections on the intervention in the methods and commented on drafts. SM was responsible for day-to-day project management, undertook practice recruitment and liaison, was involved in data collection, coding and management, drafted sections on recruitment, data collection and coding in the methods, contributed to interpretation of findings and commented on drafts. JM undertook practice recruitment and liaison, was involved in data collection, coding and management, drafted sections on data collection and coding in the methods, and commented on drafts. GMP oversaw randomisation, planned and conducted outcomes analyses, contributed to interpretation of findings, drafted sections/tables on the outcomes analyses in the methods and results, and commented on drafts. GRB conducted and interpreted the cost analyses, drafted sections on costs in the methods and results, and commented on drafts. JW was involved in the pilot study, contributed to intervention design and delivery, and commented on drafts. RH contributed to protocol design and the funding application, advised on analyses, and commented on drafts. BDWH contributed to protocol design, advised on data coding, contributed to interpretation of findings, and commented on drafts. AH commented on the funding application, advised on intervention design and delivery, and commented on drafts. DBP advised on data collection, coding and management, and commented on drafts. IH commented on protocol design, advised on analyses, and commented on drafts. AMW was a joint principal investigator. He contributed to the original protocol design and funding application, and gained ethical and regulatory approvals. He chaired the trial steering group meetings, was involved with the analysis plan, commented on drafts and edited the final paper.

Funding This study was funded by Asthma UK (Project no. 06/047). Neither the funder nor sponsor had a role in the design, data collection, analysis, interpretation, writing up, or decision to submit for publication, responsibility for which rested solely with the authors.

\section{Competing interests None.}

Ethics approval Ethics approval was provided by Norfolk Research Ethics Committee (reference number 06/00101/200).

Provenance and peer review Not commissioned; externally peer reviewed.

\section{REFERENCES}

1. British Thoracic Society \& Scottish Intercollegiate Guideline Network. British guideline on the management of asthma. Thorax 2003;58(Suppl 1):i1-94.

2. Masoli M, Fabian D, Holt S, et al. The global burden of asthma: executive summary of the GINA Dissemination Committee report. Allergy 2004;59:469-78.

3. Rabe KF, Adachi M, Lai CK, et al. Worldwide severity and control of asthma in children and adults: the global asthma insights and reality surveys. J Allergy Clin Immunol 2004;114:40-7.

4. Barnett SB, Nurmagambetov TA. Costs of asthma in the United States: 2002-2007. J Allergy Clin Immunol 2011;127:145-52.

5. Hoskins G, McCowan C, Neville RG, et al. Risk factors and costs associated with an asthma attack. Thorax 2000:55:19-24.

6. Vervloet $\mathbf{D}$, Williams AE, Lloyd $A$, et al. Costs of managing asthma as defined by a dervied Asthma Control Test (TM) score in seven European countries. Eur Respir Rev 2006:15:17-23.

7. Sturdy PM, Victor CR, Anderson HR, et al. Psychological, social and health behaviour risk factors for deaths certified as asthma: a national case-control study. Thorax 2002:57:1034-9.

8. Wainwright NW, Surtees PG, Wareham NJ, et al. Psychosocial factors and incident asthma hospital admissions in the EPIC-Norfolk cohort study. Allergy 2007:62:554-60

9. Yoon R, McKenzie DK, Miles DA, et al. Characteristics of attenders and nonattenders at an asthma education programme. Thorax 1991:46:886-90.

10. Gibson PG, Powell H, Coughlan J, et al. Self-management education and regular practitioner review for adults with asthma. Cochrane Database Syst Rev 2002;(3): CD001117.

11. Smith JR, Mugford M, Holland R, et al. Psycho-educational interventions for adults with severe or difficult asthma: a systematic review. J Asthma 2007;44:219-41.

12. Sturdy PM, Butland BK, Anderson HR, et al. Deaths certified as asthma and use of medical services: a national case-control study. Thorax 2005;60:909-15. 
13. Noble MJ, Smith JR, Windley J. A controlled retrospective pilot study of an 'at-risk asthma register' in primary care. Prim Care Respir J 2006;15:116-24.

14. NHS Employers Quality and Outcomes Framework. http://www.nhsemployers.org/ PayAndContracts/GeneralMedicalServicesContract/00F/Pages/ QualityOutcomesFramework.aspx (accessed 4 Apr 2011).

15. Communities and Local Government. The English Indices of Deprivation Report 2007. http://www.communities.gov.uk/publications/communities/ indiciesdeprivation07 (accessed 4 Apr 2011).

16. PRIMIS + Morbidity Information Query and Export Syntax (MIOUEST) Software. http://www.primis.nhs.uk/index.php/services/information-services/miquest (accessed 4 Apr 2011).

17. Tattersfield AE, Postma DS, Barnes PJ, et al. Exacerbations of asthma: a descriptive study of 425 severe exacerbations. The FACET International Study Group. Am J Respir Crit Care Med 1999;160:594-9.

18. Charlson ME, Pompei P, Ales KL, et al. A new method of classifying prognostic comorbidity in longitudinal studies: development and validation. J Chronic Dis 1987; 40:373-83.

19. Campbell MK, Mollison J, Grimshaw JM. Cluster trials in implementation research: estimation of intracluster correlation coefficients and sample size. Stat Med 2001;20:391-9

20. Altman DG, Dore CJ. Baseline comparisons in randomized clinical trials. Stat Med 1991;10:797-9.

21. Donner A, Klar N. Design and Analysis of Cluster Randomization Trials in Health Research. London: Arnold, 2000.

22. Curtis L. Unit Costs of Health and Social Care 2008. Canterbury: University of Kent Personal Social Services Research Unit, 2008.

23. Department of Health. National Schedule of Reference Costs 2007-08. London: Department of Health, 2009.
24. British Medical Association/Royal Pharmaceutical Society of Great Britain British National Formulary (BNF) 55. London: Pharmaceutical Press, 2008.

25. The NHS Information Centre for Health and Social Care. Prescription Cost Analysis: England 2008. http://www.ic.nhs.uk/statistics-and-data-collections/ primary-care/prescriptions/prescription-cost-analysis-2008 (accessed 4 Apr 2011).

26. Bachmann Mo, Fairall $L$, Clark $A$, et al. Methods for analyzing cost effectiveness data from cluster randomized trials. Cost Eff Resour Alloc 2007;5:12.

27. Reddel HK, Taylor DR, Bateman ED, et al. An official American Thoracic Society/ European Respiratory Society statement: asthma control and exacerbations: standardizing endpoints for clinical asthma trials and clinical practice. Am J Respir Crit Care Med 2009;180:59-99.

28. Crane J, Pearce N, Burgess C, et al. Markers of risk of asthma death or readmission in the 12 months following a hospital admission for asthma. Int $\mathrm{J}$ Epidemiol 1992:21:737-44.

29. Mitchell EA, Bland JM, Thompson JM. Risk factors for readmission to hospital for asthma in childhood. Thorax 1994;49:33-6.

30. Boyd M, Lasserson TJ, McKean MC, et al. Interventions for educating children who are at risk of asthma-related emergency department attendance. Cochrane Database Syst Rev 2009;(2):CD001290.

31. Tapp S, Lasserson TJ, Rowe B. Education interventions for adults who attend the emergency room for acute asthma. Cochrane Database Syst Rev 2007; (3):CD003000.

32. Lemmens KM, Nieboer AP, Huijsman R. A systematic review of integrated use of disease-management interventions in asthma and COPD. Respir Med 2009;103:670-91.

33. Griffiths C, Foster G, Barnes N, et al. Specialist nurse intervention to reduce unscheduled asthma care in a deprived multiethnic area: the east London randomised controlled trial for high risk asthma (ELECTRA). BMJ 2004;328:144. 